\title{
Zusammensetzung der Lipoproteinfraktionen VLDL, LDL und HDL bei Hyperlipämikern der Typen IIa, IIb, III, IV und V im Vergleich zu Stoffwechselgesunden
}

\author{
Von V. Hutt, H. U. Klör, J. G. Wechsler und H. Ditschuneit \\ Dept. Innere Medizin, Abt. Stoffwechsel und Ernährungswissenschaften der Universität Ulm
}

(Eingegangen am 29. März/5. Oktober 1983)

Zusammenfassung: Die chemische Zusammensetzung der LipoproteinfraktionenVLDL, LDL und HDL wurde an 82 Patienten mit primären Hyperlipoproteinämien (37 Typ IIa, 7 Typ IIb, 3 Typ III, 25 Typ IV, 10 Typ V) und 10 Stoffwechselgesunden näher untersucht. Die Lipoproteinfraktionen wurden mit Hilfe der präparativen Ultrazentrifugation gewonnen. Jede Fraktion wurde auf ihren Gehalt an Cholesterin, Triglyceriden, Phospholipiden und Protein analysiert.

Unterschiede in der Zusammensetzung der Lipoproteine konnten in allen 3 Fraktionen sowohl im Vergleich zur Gruppe der Stoffwechselgesunden als auch innerhalb der einzelnen Hyperlipoproteinämietypen beobachtet werden.

Composition of VLDL, LDL and $H D L$ lipoprotein fractions in type IIa, IIb, III, IV and V hyperlipaemic patients and in normal individuals

Summary: The chemical composition of VLDL, LDL and HDL was studied in 82 patients with primary hyperlipoproteinaemia (37 type IIa, 7 type IIb, 3 type II, 25 type IV, 10 type V) and in ten metabolically normal individuals. Lipoprotein fractions were prepared by preparative ultracentrifugation. Each fraction was analysed for cholesterol, triglycerides, phospholipids and protein.

Differences between patients and normal individuals, and between the individual types of hyperlipoproteinaemia were evident in the composition of all three lipoprotein fractions.

\section{Einfuihrung}

Die primären Hyperlipoproteinämien lassen sich nach Empfehlungen der Weltgesundheitsorganisation (WHO) in zumindest 6 verschiedene Formen einteilen, die Hyperlipoproteinämietypen I, IIa, IIb, III, IV und V (1). Bei der Einteilung wird dabei vor allem der Gehalt an Triglyceriden in den Very-LowDensity-Lipoproteinen (VLDL) und an Cholesterin in den Low-Density-Lipoproteinen (LDL) berücksichtigt. Die High-Density Lipoproteine (HDL) spielen bei dieser Klassifizierung keine Rolle.

Hohe LDL-Cholesterinkonzentrationen gelten als wesentlicher Risikofaktor für die Entstehung der
Atherosklerose $(2,3)$. Im Gegensatz dazu sind die Meinungen hinsichtlich der Bedeutung erhöhter Triglyceridwerte, normalerweise durch eine Vermehrung der VLDL-Lipoproteine bzw. der VLDL-Triglyceride gekennzeichnet, als Risikofaktor geteilt (4, 5). In zahlreichen Untersuchungen der letzten Jahre konnte dagegen eine negative Korrelation zwischen HDL-Lipoproteinen bzw. HDL-Cholesterin einerseits und der koronaren Herzerkrankung andererseits festgestellt werden $(6,7,8)$.

Zahlreiche Lipoproteinuntersuchungen beschränken sich deshalb auf die Bestimmung des Cholesterins und der Triglyceride in den 3 Lipoproteinfraktionen 
VLDL, LDL und HDL. Am Aufbau der Lipoproteinpartikel sind aber neben Cholesterin und Triglyceriden auch Phospholipide und Proteine - Apolipoproteine genannt - beteiligt. Dabei bilden die mehr hydrophilen Phospholipide, Apolipoproteine und freies Cholesterin die äußere Hülle dieser zumeist kugelförmigen Gebilde, während sich die hydrophoben Triglyceride und Cholesterinester im Kern anordnen.

Im Rahmen dieser Studie sollte deshalb die Verteilung von Cholesterin, Triglyceriden, Phospholipiden und Protein in den Lipoproteinfraktionen VLDL, LDL und HDL bei Hyperlipämikern der Typen IIa, IIb, III, IV, V und bei einer Gruppe Stoffwechselgesunder näher untersucht werden.

\section{Material und Methodik}

\section{Patienten}

82 Patienten mit primären Hyperlipoproteinämien und 10 Stoffwechselgesunde wurden in die Studie aufgenommen. Der Großteil der Patienten war noch nie medikamentös behandelt worden; bei den restlichen Hyperlipämikern lag die letzte medikamentöse Behandlung schon über 3 Monate zurück. Die Typisierung der Hyperlipoproteinämie wurde anhand der Cholesterin- und Triglyceridwerte im Plasma sowie der Lipidelektrophorese (9) nach Kriterien der WHO (1) ermittelt. Für eine exakte Festlegung des Hyperlipoproteinämietyps III wurden zusätzlich Empfehlungen von Hazzard et al. (10) und Fredrickson et al. (11) berücksichtigt. Von den 82 Patienten hatten 37 Patienten eine Hyperlipoproteinämie vom Typ IIa; bei 7 Patienten lag eine Hyperlipoproteinämie vom Typ IIb, bei 3 Patienten ein Typ III, bei $25 \mathrm{~Pa}-$ tienten ein Typ IV und bei 10 Patienten ein Typ V nach Fredrickson vor. In der Gruppe der Stoffwechselgesunden wurden solche Personen zusammengefaßt, die im Plasma Werte von kleiner als
$6,5 \mathrm{mmol} / /$ für Gesamt-Cholesterin und kleiner als $1,5 \mathrm{mmol} / / \mathrm{für}$ Gesamt-Triglyceride vorweisen konnten. Die wichtigsten persönlichen Daten der Patienten sind in Tabelle 1 zusammengefaßt.

\section{Verfahren}

Nach jeweils mindestens 12stündiger Nahrunígskarenz erfolgten die Blutentnahmen. Zur Bestimmung der Lipoproteinfraktionen VLDL, LDL und HDL wurde die Ultrazentrifugation (UZ) nach Empfehlungen von Havel et al. (12) durchgeführt. Die einzelnen UZ-Fraktionen wurden anschließend durch Pipettieren abgetrennt. Verluste während der ganzen UZ-Prozedur und während des Abpipettierens sind unvermeidbar. Durch experimentelle Bestimmung der Wiederfindungsraten der verschiedenen Lipoproteinklassen konnte der Verlust quantifiziert werden. Die Wiederfindungsrate der VLDL betrug $86 \%$, der LDL $75 \%$ und der HDL $65 \%$ und wurde bei der Berechnung der angegebenen Konzentrationen berücksichtigt. Die Triglyceride wurden nach Eggstein \& Kreutz (13), die Phospholipide nach Zilversmit \& Davis (14), Gesamtcholesterin, freies- und verestertes Cholesterin nach Röschlau et al. (15) bestimmt. Die Cholesterinestermasse wurde durch Multiplikation der Masse an verestertem Cholesterin mit dem Faktor 1,67 ermittelt und zur Berechnung des relativen Anteils des Gesamtcholesterins hinzugezogen. Dadurch wird der Massenanteil der Cholesterinesterfettsäuren berücksichtigt (16). Der Proteingehalt der Fraktionen wurde nach der Methode von Lowry et al. (17) ermittelt. Als Standard diente Rinderserumalbuminlösung $(1 \mathrm{~g} / 1)$. Nach der Farbentwicklung wurden die zu untersuchenden Proben mit Diethylether ausgeschüttelt, um Trübungseffekte, die vor allem bei stark lipämischen Proben ausgeprägt sind, auszuschließen. Eine mögliche Verunreinigung der Lipoproteinproben mit Albumin wurde qualitativ mit Hilfe des Ouchterlony-Tests überprüft (18). Quantitativ wurde der Albuminanteil mit der von Mancini (19) entwickelten Radialimmundiffusionstechnik ermittelt und bei der Angabe der Proteinkonzentrationen berücksichtigt. Die Reinheit der Lipoproteinfraktionen wurde lipidelektrophoretisch überprüft (9).

Als statistisches Prüfverfahren wurde der Student=t-Test angewandt. Als statistsch nicht signifikant wurde ein Grenzwert von $p$ $>0,05$ festgelegt.

Tab. 1. Daten der untersuchten Patienten $(\bar{x} \pm S E M)$.

\begin{tabular}{|c|c|c|c|c|c|c|c|c|}
\hline \multirow{2}{*}{$\begin{array}{l}\text { Hyper- } \\
\text { lipo- } \\
\text { protein- } \\
\text { ämie- } \\
\text { Typ }\end{array}$} & \multicolumn{2}{|l|}{ Patienten } & \multirow{2}{*}{$\begin{array}{l}\text { Alter } \\
\text { (Jahre) }\end{array}$} & \multirow{2}{*}{$\begin{array}{l}\text { Körpergewicht } \\
\text { (kg) }\end{array}$} & \multirow{2}{*}{$\begin{array}{l}\text { Körpergröße } \\
\text { (cm) }\end{array}$} & \multirow{2}{*}{$\begin{array}{l}\text { Gesamt- } \\
\text { cholesterin } \\
\text { im Plasma } \\
(\mathrm{mmol} / \mathrm{l})\end{array}$} & \multirow{2}{*}{$\begin{array}{l}\text { Triglyceride } \\
\text { im Plasma } \\
(\mathrm{mmol} / \mathrm{l})\end{array}$} & \multirow{2}{*}{$\begin{array}{l}\text { Phospho- } \\
\text { lipide } \\
\text { im Plasma } \\
\text { (mmol/l) }\end{array}$} \\
\hline & Gesamt & $\begin{array}{l}\text { nach } \\
\text { Geschlecht }\end{array}$ & & & & & & \\
\hline IIa & 37 & $\begin{array}{l}22 \% \\
15 \delta\end{array}$ & $50,1 \pm 2,9$ & $68,4 \pm 3,9$ & $163 \pm 2,6$ & $8,76 \pm 0,4$ & $1,59 \pm 0,1$ & $4,22 \pm 0,1$ \\
\hline IIb & 7 & $\begin{array}{l}5 \% \\
2 \%\end{array}$ & $58,4 \pm 6,2$ & $65,8 \pm 4,2$ & $167 \pm 1,3$ & $7,34 \pm 0,4$ & $2,73 \pm 0,4$ & $3,82 \pm 0,2$ \\
\hline III & 3 & $\begin{array}{l}1 \% \\
28\end{array}$ & $61,0 \pm 3,2$ & $70,8 \pm 4,2$ & $163 \pm 7,3$ & $7,96 \pm 0,8$ & $3,72 \pm 0,5$ & $4,10 \pm 0,1$ \\
\hline IV & 25 & $\begin{array}{r}8 \% \\
17 \%\end{array}$ & $52,6 \pm 3,9$ & $74,8 \pm 3,9$ & $165 \pm 2,9$ & $6,00 \pm 0,2$ & $3,36 \pm 0,2$ & $3,65 \pm 0,1$ \\
\hline$s$ & 10 & $\begin{array}{l}6 \% \\
4 \delta\end{array}$ & $64,4 \pm 3,8$ & $74,1 \pm 6,4$ & $162 \pm 3,9$ & $6,23 \pm 0,3$ & $10,04 \pm 2,0$ & $4,95 \pm 0,6$ \\
\hline $\begin{array}{l}\text { Stoff- } \\
\text { wechsel- } \\
\text { gesunde }\end{array}$ & 10 & $\begin{array}{l}6 \% \\
48\end{array}$ & $32,1 \pm 3,8$ & $62,2 \pm 3,8$ & $170 \pm 3,3^{\circ}$ & $4,79 \pm 0,2$ & $0,90 \pm 0,1$ & $2,89 \pm 0,1$ \\
\hline
\end{tabular}




\section{Ergebnisse}

In den Abbildungen 1-3 sind die Befunde graphisch zusammengefaßt.

\section{VLDL}

In der VLDL-Fraktion (Abb. 1) läßt sich eine signifikante Erhöhung des Triglyceridanteils bei den Typen IV und V mit 0,551 und 0,526 im Vergleich zur Kontrollgruppe mit 0,458 nachweisen. Bei den Typen IIa und III liegen die entsprechenden Werte mit 0,443 und 0,400 niedriger; beim Typ IIb beträgt der Triglyceridanteil 0,506. Alle diese Unterschiede waren jedoch statistisch nicht signifikant. Das Gesamtcholesterin ist beim Typ IV mit 0,199 nur geringfügig, bei den Typen Ila, III und V mit 0,244, 0,322 und 0,238 signifikant erhöht. Der Phospholipidanteil ist bei den Typen IIb, IV und V mit 0,171, 0,169 bzw. 0,149 signifikant niedriger als bei der Gruppe der Stoffwechselgesunden mit 0,210. Die Phospholipidkonzentration beim Typ IIa bzw. III beträgt 0,208 bzw. 0,187 . Der Proteinanteil ist bei allen $\mathrm{Hy}-$ perlipoproteinämie-Typen im Vergleich zur Kontrolle $(0,138)$ signifikant vermindert. Im Einzelnen beträgt der Proteinanteil bei den Typen IIa-V $0,105,0,086,0,092,0,082$ und 0,087 .

\section{LDL}

In der LDL-Fraktion (Abb. 2) ist der relative Proteinanteil mit rund 0,200 bei allen Hyperlipoproteinämie-Typen und der Gruppe der Stoffwechsel-
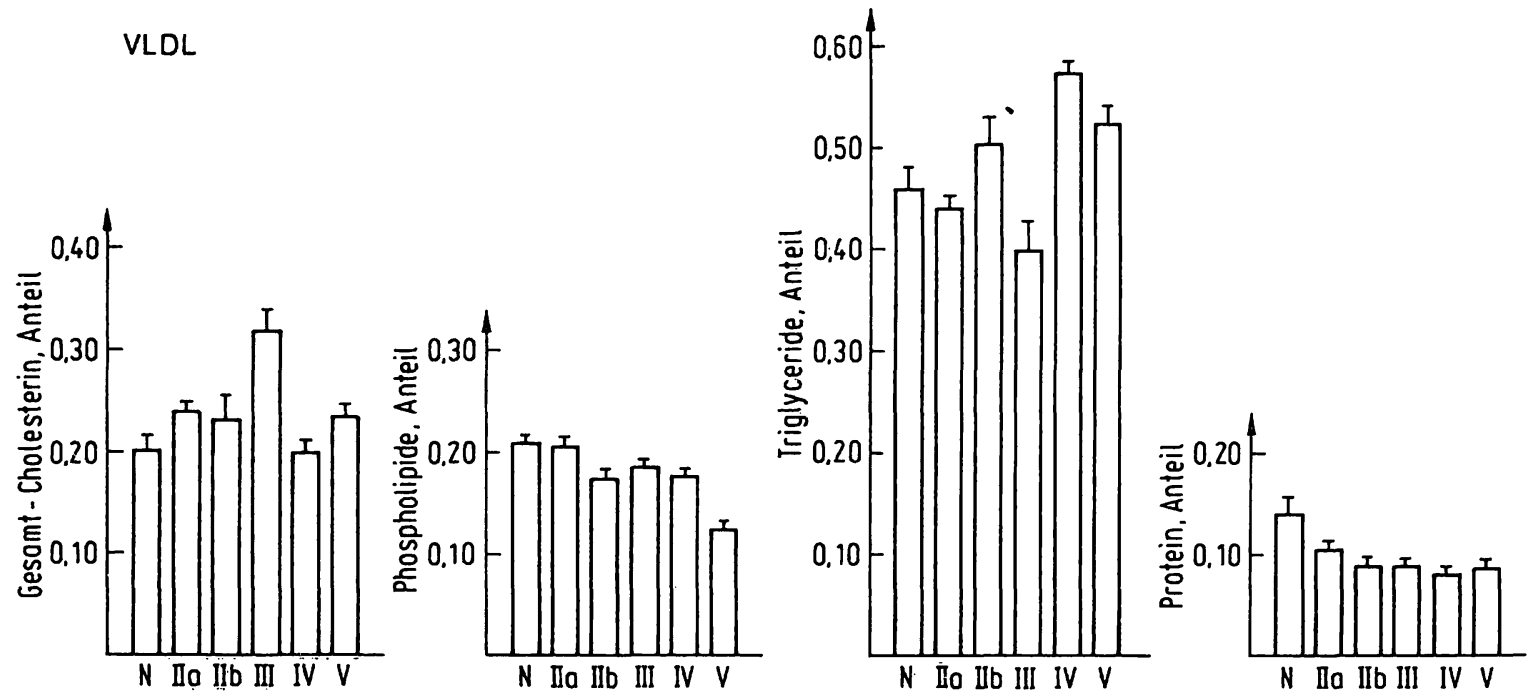

Abb. 1. Zusammensetzung der VLDL-Lipoproteinfraktion ( $<1,006 \mathrm{~kg} / / \mathrm{l})$ bei Hyperlipämikern der Typen Ila, IIb, III, IV, V und bei Stoffwechselgesunden $(N)(\bar{x} \pm S E M$, ausführliche Erläuterungen s. Text).
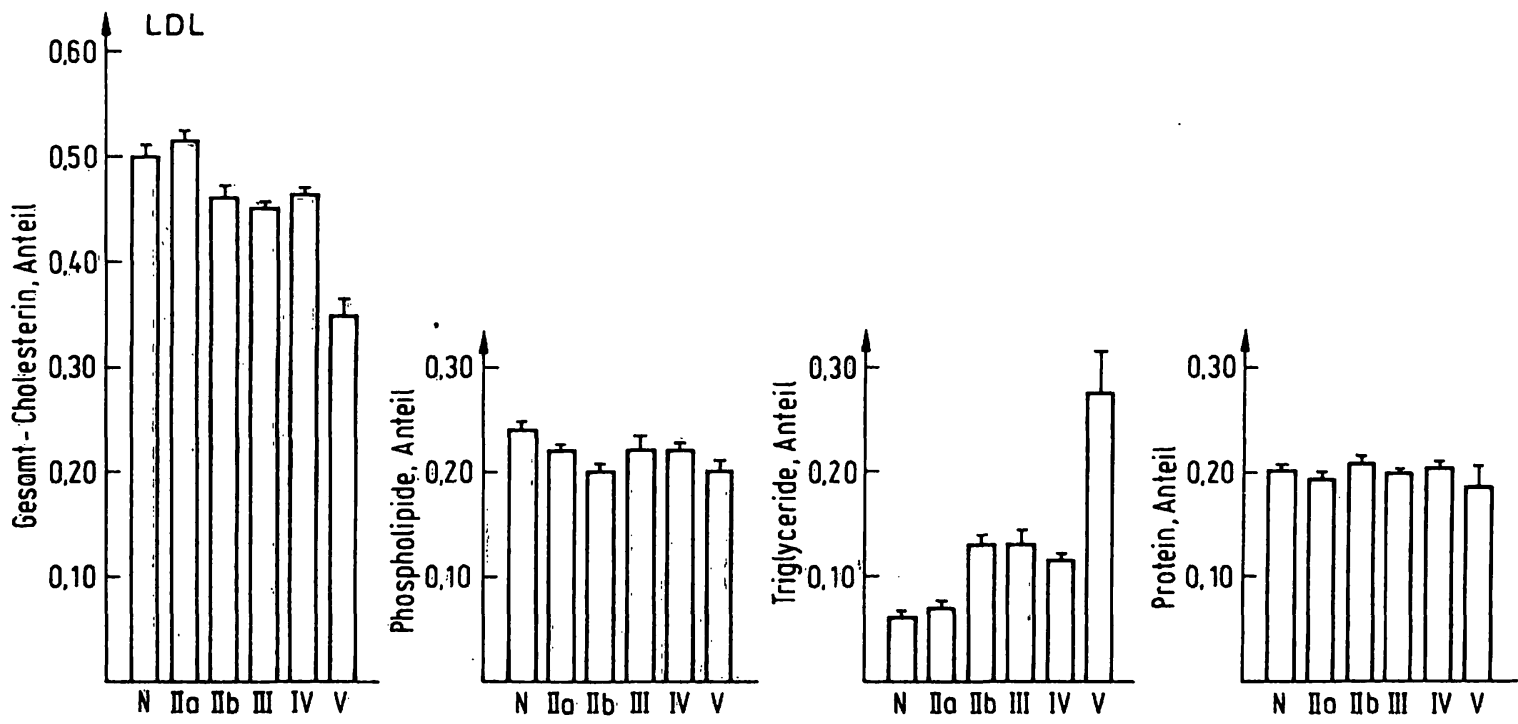

Abb. 2. Zusammensetzung der LDL-Lipoproteinfraktion $(\mathrm{d}=1,006-1,063 \mathrm{~kg} / \mathrm{l})$ bei Hyperlipänikern der Typen IIa, IIb. III, IV, V und bei Stoffwechselgesunden $(N)(\bar{x} \pm S E M$, ausführliche Erläuterungen $s$. Text). 
gesunden nahezu identisch. Der höchste Wert wurde beim Typ Ilb mit 0,207, der niedrigste beim Typ V mit 0,185 gefunden. Abweichungen in größerem Maße zeigen sich jedoch im Triglycerid- und Cholesteringehalt. Der Triglyceridanteil ist bei den Typen IIb, III, IV und V mit $0,127,0,130,0,115$ und 0,274 signilikant höher als bci der Gruppe der Stoffwechselgesunden mit 0,060 ; beim Typ IIa beträgt cr 0,(070. Dagegen ist der Cholesterinanteil bei den Typen IIb-V mit $0,460,0,450,0,462$ und 0,345 signifikant niedriger als beim Referenzkollektiv mit 0,501 . Im Cholesteringehalt vergleichbar mit den Stoffwechselgesunden ist der Typ Ila mit 0,514. Eine signifikante Verringerung an Phospholipiden gegenüber der Kontrollgruppe $(0,239)$ ist bei den Typen Ila $(0,221)$, IIb $(0,207)$, IV $(0,221)$ und V $(0,196)$ zu beobachten.

\section{HDL}

Der Triglyceridgehalt in der HDL-Fraktion (Abb. 3) ist bei den Typen Ila $(0,068)$, IIb $(0,077)$, IV $(0,084)$ und $V(0,125)$ statistisch signifikant höher als bei der Kontrollgruppe $(0,049)$. Im Cholesterin ist dagegen ein signifikanter Unterschied gegenüber der Gruppe der Stoffwechselgesunden $(0,263)$ nur bcim Typ V $(0,207)$ zu beobachten. Bei den Typen Ila und IIb liegen die entsprechenden Werte mit 0,289 und 0,285 geringfügig niedriger. Ein signifikanter Unterschied in den Phospholipiden zeigt sich zwischen den Stoffwechselgesunden $(0,265)$ und den Typen IIa $(0,237)$ und IIb $(0,234)$. Der Proteinanteil ist bei den Typen IIa $(0,405)$, Ilb $(0,403)$, III $(0,422)$ und IV $(0,417)$ niederer im Vergleich zur Kontrollgruppe mit 0,423. Beim Typ $V$ liegt der entsprechende Wert mit 0,439 höher. Alle Unterschiede im Proteingehalt waren jedoch statistisch signifikant nicht zu sichern.
Auch innerhalb der einzelnen Hyperlipoproteinämie-Typen sind zahlreiche signifikante Unterschiede im Cholesterin-, Triglycerid-, Phospholipidund Proteinanteil in den 3 Lipoproteinfraktionen zu beobachten, die in Tabelle 2 zusammengefaßt sind.

Tab. 2. Statistisch signifikante Unterschiede innerhalb der $\mathbf{H y -}$ perlipoproteinämic-Typen IIa, IIb, III, IV und V im Cholesterin-, Triglycerid-, Phospholipid- und Proteinantcil in den 3 Lipoproteinfraktionen VLDL, LDL und HDL $(\mathrm{p}<0,05)$.

\begin{tabular}{lllll}
\hline & $\begin{array}{l}\text { Gcsamt- } \\
\text { Cholesterin }\end{array}$ & Triglyceride & $\begin{array}{l}\text { Phospho- } \\
\text { lipide }\end{array}$ & Protein \\
\hline VLDL & IIa $\rightarrow$ III & IIa $\rightarrow$ IIb & IIa $\rightarrow$ IIb & IIa $\rightarrow$ IIb \\
& IIa $\rightarrow$ IV & IIa $\rightarrow$ IV & IIa $\rightarrow$ IV & IIa $\rightarrow$ IV \\
& IIb $\rightarrow$ IV & IIa $\rightarrow$ V & IIa $\rightarrow$ V & IIa $\rightarrow$ V \\
& III $\rightarrow$ IV & III $\rightarrow$ IV & IV $\rightarrow$ V & \\
& III $\rightarrow$ V & III $\rightarrow$ V & & \\
& IV $\rightarrow$ V & IV $\rightarrow$ V & & \\
LDL & IIa $\rightarrow$ IIb & IIa $\rightarrow$ IIb & IIa $\rightarrow$ V & \\
& IIa $\rightarrow$ IV & IIa $\rightarrow$ III & IV $\rightarrow$ V & \\
& IIa $\rightarrow$ V & IIa $\rightarrow$ IV & & \\
& IIb $\rightarrow$ V & IIa $\rightarrow$ V & & \\
& IV $\rightarrow$ V & II $\rightarrow$ V & & IIa $\rightarrow$ V \\
& & IV $\rightarrow$ V & & IIb $\rightarrow$ V \\
HDL & IIa $\rightarrow$ IV & IIa $\rightarrow$ IV & & \\
& IIa $\rightarrow$ V & IIa $\rightarrow$ V & & \\
& IIb $\rightarrow$ V & IV $\rightarrow$ V & & \\
& IV $\rightarrow$ V & & & \\
\hline
\end{tabular}

\section{Discussion}

Die Untersuchungsergebnisse offenbaren zahlreiche signifikante Unterschiede im Anteil von Cholesterin, Triglyceriden, Phospholipiden und Protein an den Lipoproteinpartikeln der 3 Lipoproteinfraktionen VLDL, LDL und HDL bei den Hyperlipoproteinämie-Typen IIa, IIb, III, IV und V im Vergleich zu
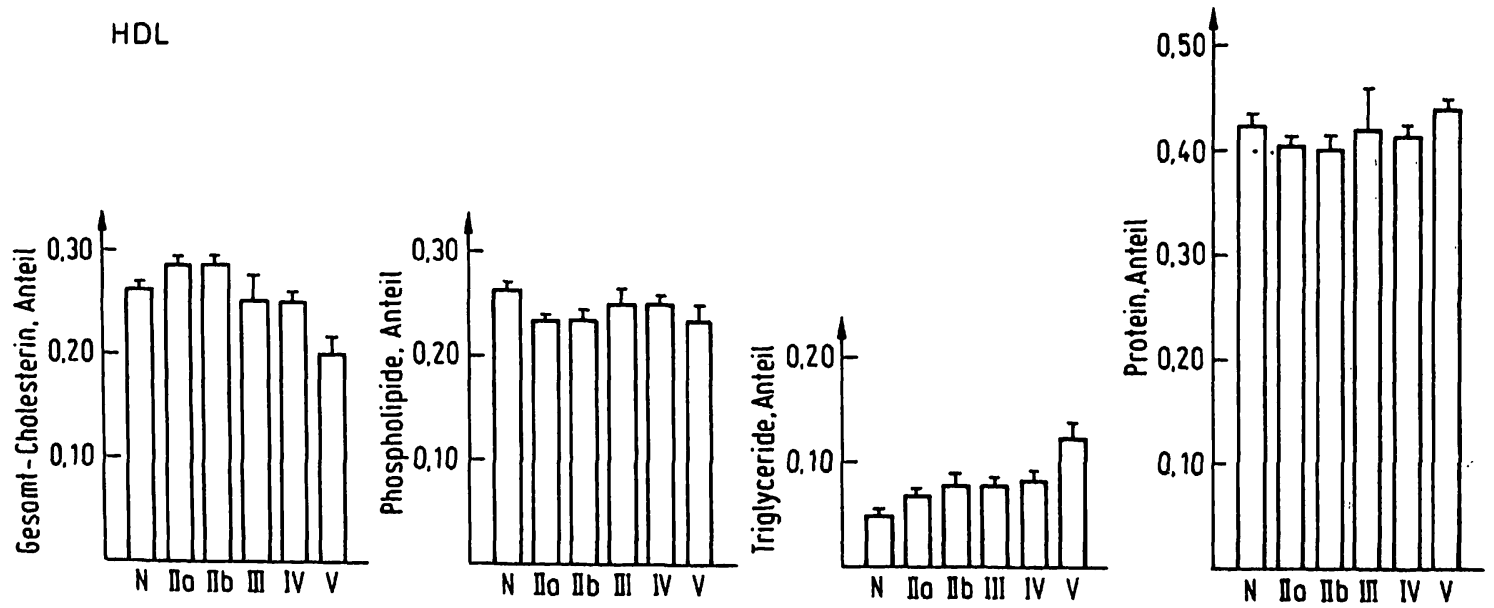

Abb. 3. Zusammensetzung der HDL-Lipoproteinfraktion $(d=1,063-1,25 \mathrm{~kg} / 1)$ bei Hyperlipämikern der Typen IIa; Ilb, III, IV, V und hei Stoffwechselgesunden $(N)(\bar{x} \pm S E M$, ausführliche Erläuterungen s. Text). 
Stoffwechselgesunden. So konnten in der VLDLDichteklasse signifikante Unterschiede im Proteingehalt aller Hyperlipoproteinämie-Typen gegenüber der Kontrolle nachgewiesen werden. Der VLDLCholesterinanteil war bei den Typen IIa, III und V signifikant erhöht; eine signifikante Zunahme im Triglyceridanteil konnte bei den Typen IV und V beobachtet werden. Der Cholesterinanstieg beim Typ III läßt sich durch eine Anhäufung von ,intermediate density lipoproteins" (IDL) erklären. In der LDL-Dichteklasse zeigte sich gegenüber der Kontrolle eine signifikanter Anstieg im Triglycerid bei den Typen IIb, III, IV und V bei einem gleichzeitigen signifikanten Abfall des Cholesterins bei diesen Hyperlipoproteinämie-Formen. Die LDL-Phospholipide waren bei allen HyperlipoproteinämieTypen mit Ausnahme des Typs III signifikant niedriger. In der HDL-Dichteklasse ist die signifikante Triglyceridanreicherung der Lipoproteinpartikel bei den Typen IIa, IIb, IV und V am auffallendsten.

Gegenüber Stoffwechselgesunden weisen die Lipoproteinfraktionen bei den verschiedenen Hyperlipoproteinämie-Formen somit eine deutlich andere chemische Zusammensetzung auf, d.h. sie sind pathologisch verändert. Bei den verschiedenen Hyperlipoproteinämie-Typen handelt es sich deshalb im Vergleich zu Stoffwechselgesunden nicht nur um eine Vermehrung gleicher Lipoproteinpartikel in einer oder mehrerer Lipoproteinfraktionen über das normale Maß hinaus.

Auch in der Literatưr gibt es Hinweise dafür (2025), daß zumindest einzelne Lipoproteinklassen bei Hyperlipämikern im Vergleich zu einer gesunden Kontrollgruppe eine pathologische Zusammensetzung aufweisen. Bestärkt werden diese Vermutungen durch spezielle Untersuchungen die an LDLFraktionen der Typ II Hyperlipoproteinämien vorgenommen wurden. Elektronenoptische Aufnahmen zeigen, daß die LDL-Partikel des Typs IIa nicht wie bei Gesunden eine sphärische Form haben, sondern dazu neigen, eine mehr ellipsoide Form anzunehmen. Auch die Aggregationsform der Typ II-LDL weicht von der Norm ab; sie lagern sich in Form von geraden oder verzweigten Ketten aneinander (26). Des weiteren konnte nachgewiesen werden, daß bei Heterozygoten vom Hyperlipoproteinämie-Typ II die Dielektrizitätskonstante einer LDL-Emulsion niederer ist als bei Gesunden und bei Homozygoten wiederum niedriger ist als bei Heterozygoten (27). Ferner wird über eine Erhöhung des Molekulargewichts (28) und des isoclektrischen Punktes (29) der LDL von Patienten de:; 'Typs II gegenüber gesunden Probanden berichtet.

Auch innerhalb der einzelnen Hyperlipoproteinämie-Typen sind zum Teil beträchtliche Unterschiede in der Zusammensetzung der 3 Lipoproteinfraktionen festzustellen. So zeichnet sich der Typ IIb im Vergleich zum Typ Ila durch eine signifikante Erhöhung im Triglyceridgehalt der VLDL und LDL aus. Dagegen ist die Cholesterinkonzentration der LDL-Partikel beim Typ IIa signifikant höher. Aufgrund von Untersuchungen der letzten Jahre kann die Typ Ilb Hyperlipoproteinämie nicht als Untergruppe des Typs IIa angesehen werden. Der Typ IIb stellt als sogenannte "combined hyperlipoproteinemia" eine eigenständige Erkrankung dar $(30,31)$. Beim Typ III sei, im Gegensatz zu allen anderen Hyperlipoproteinämie-Typen, die extreme Cholesterinanreicherung in den VLDL-Partikeln hervorgehoben. Als Folge davon ist der Gehalt an Triglyceriden in den VLDL auffallend niedrig im Unterschied zu den anderen Hyperlipoproteinämieformen. Die Typen IV und $V$ heben sich von den anderen durch ihren hohen Triglyceridanteil in den VLDL-Partikeln ab. In weit geringerem Maße sind zwischen den verschiedenen Hyperlipoproteinämie-Typen signifikante Unterschiede im Protein- und Phospholipidgehalt in den einzelnen Lipoproteinfraktionen zu beobachten. Es kann deshalb spekuliert werden, daß die Abweichungen in der Lipoproteinzusammensetzung der Hyperlipoproteinämie-Typen hauptsächlich durch einen unterschiedlichen Anteil an Kernmaterialbestandteilen verursacht wird. Unterschiede in der Oberflächenbeschaffenheit der Lipoproteinpartikel scheinen möglicherweise weit weniger ausgeprägt.

Die Befunde berechtigen zu der Annahme, daß den verschiedenen Hyperlipoproteinämie-Formen kein einheitlicher Stoffwechseldefekt zugrunde liegt, sondern vielmehr verschiedenartige Stoffwechselstörungen für die einzelnen Hyperlipoproteinämie-Formen verantwortlich sind. Mögliche vorliegende Störungen bei den verschiedenen HyperlipoproteinämieTypen werden in der Literatur (32-40) ausführlich diskutiert. 


\section{Literatur}

1. Beaumont, J. L., Carison, L. A., Cooper, G. R., Fejfer, Z., Frederickson, D. S. \& Strasser, T. (1970) World Health Organ. 43, 891-908.

2. Kannel, W. B.. Castelli, W. P., Gordon, F. \& McNamara, P. (1971) Ann. Int. Med. 74, 1-12.

3. Gofman, J. W., Lindgren, F. T., Elliot, H., Mantz, W., Hewitt, J., Strisower, B. \& Herring, V. (1950) Science 111, $166-186$.

4. Carlson, L. A. \& Böttiger, L. E. (1972) Lancet $1,865-868$.

5. Hulley, S. B., Roseman, R. H., Bawol, R. D. \& Brand, R. J. (1980) New Engl. J. Med. 302, 1383-1389.

6. Castelli, W. P., Doyle, J. T. \& Gordon, T. (1977) Circulation 55, 767-772.

7. Miller, G. J. \& Miller, N. E. (1975) Lancet $I, 16-19$.

8. Gordon, T., Castelli, W. P., Hjortland, M. C., Kannet, W. B. \& Dawber, T. R. (1977) Am. J. Med. 62, 707-714.

9. Rapp. W. \& Kahlke, W. (1968) Clin. Chim. Acta 19, 493498.

10. Hazzard, W., Porte, D. Jr. \& Biermann, E. L. (1972) Metabolism 21, 1009-1019.

11. Fredrickson, D. S., Morganroth, J. \& Lewy, R. I. (1975) Ann. Intern. Med. 82, 150-157.

12. Havel, R. J., Eder, H. A. \& Bragdon, H. J. (1955) J. Clin. Invest. 34. 1345-1353.

13. Eggstein. M. \& Kreutz, F. H. (1966) Klin. Wochenschr. 44, 262-267.

14. Zilversmit, D. B. \& Davis. A. K. (1950) J. Lab. Clin. Med. $35,155-160$.

15. Röschlau, P., Bernt, E. \& Gruber, W. (1974) Z. Klin. Chem. Klin. Biochem. 12, 403-407.

16. Bragdon, J. H., Eder, H. A., Gould, R. G. \& Havel, R. J. (1956) Circulation Res. 4, 129.

17. Lowry, H. O., Rosebrough, N. J., Farr, A. L. \& Randall, R. J. (1951) J. Biol. Chem. 193, 265-275.

18. Ouchterlony, O. (1958) Allergy 5, 1-78.

19. Mancini, G., Carbonara, A. O. \& Heremaus, J. F. (1965) Immunochemistry 2, 235-254.
20. Bagnall, T. F. \& Lloyd, J. K. (1975) Clin. Chim. Acta 59, $271-276$.

21. Carlson, L. A. (1976). Lipoprotein Metabolism, Springer Verlag Berlin, Heidelberg, New York. 69-79.

22. Carlson, L. A. (1974) Adv. Exp. Med. Biol. 63, 185-199.

23. Haacke, H., Parwaresch, M. R. \& Mädè, Ch. (1975) Klin. Wochenschr. 53, 1121-1124.

24. Simons, L. A. \& Williams, P. F. (1975) Clin. Chim. Acta 61. $341-352$.

25. Weisweiler, P. \& Schwandt; P. (1978) Atherosclerosis $3 I$, 53-58.

26. Agostini, B. (1973) Naturwissenschaften 60, 111.

27. Grant, E., Sheppard, R. J., Mills, G. L. \& Stock, J. (1972) Lancet $I, 1159-1161$.

28. Fisher, U. R. (1972) Ann. Clin. Lab. Sci. 2, 198-202.

29. Ghosh, S., Basu, M. K. \& Schweppe, J. S. (1973) Proc. Exp. Biol. Med. 142, 1322-1325.

30. Glueck, C. J., Fallat, R., Buncher, C. R. \& Steiner, P. (1973) Metabolism 22, 1403-1428.

31. Rose, H. K., Kranz, P., Weinstock, M., Juliano, I. \& Haft, J. I. (1974) Atherosclerosis 20, 51-64.

32. Langer, T., Strober, W. \& Levy, R. I. (1972) J. Clin. Invest. $51,1528-1536$.

33. Brown, M. S. \& Goldstein, J. L. (197̄4) Proc. Nat. Acad. Sci. USA $71,788-792$.

34. Brown, M. S. \& Goldstein, J. L. (1975) Cell 6, 307-316.

35. Chait, A., Brunzell, J. D., Albers, J. J. \& Hazzard, W. R. (1976) Lancet $I, 1176-1178$.

36. Sailer, S., Sandhofer, F. \& Braunsteiner, H. (1966) Klin. Wochenschr. 44, 1032-1036.

37. Havel, R. J., Kane, J. P., Balasse, E. O., Segel, N. \& Basso, L. V. (1970) J. Clin. Invest. 49, 2017-2034.

38. Reaven, G. M., Hill, D. B., Gross, C. \& Farquhar, J. W. (1965) J. Clin. Invest. 44, 1826-1833.

39. Adams, P. W., Kissebah, A. H., Harrigan, P., Stokes, T. \& Wynn, V. (1974) J. Clin. Invest. 4, 149-162.

40. Glueck, C. J. (1971) Metabolism 20, 691-712.

\section{Dr. V. Hutt}

Abt. Innere Medizin II

Universität Ulm

Steinhövelstr. 9

D-7900 Ulm 